\title{
Dicotomía Naturaleza - Tecnología: diálogo entre el Constructivismo Social de la Tecnología y la Ecología Política Latinoamericana
}

\author{
Nature - Technology dichotomy: dialogue between Social Construction of \\ Technology and Latin American Political Ecology
}

\author{
Ayelen Cavalli' \\ Tomás Javier Carrozza ${ }^{2}$
}

\section{Resumen}

En el presente artículo se abordará la relación entre Tecnología y Naturaleza, dentro del Constructivismo Social de la Tecnología (SCOT) y se indagaran los posibles argumentos que eviten recaer tanto en el determinismo tecnológico como en el determinismo social. En segundo lugar, esta investigación se propone tomar las herramientas provenientes de la Ecología Política Latinoamericana (EPL), focalizando en los desarrollos realizados a partir de los denominados "giro descolonial" en los estudios latinoamericanos y "giro ontológico" dentro de la antropología, los cuales confluyen en el trabajo de Arturo Escobar, para reflexionar sobre las posibilidades que ofrecen las mismas para una lectura descolonial de la dicotomía Tecnología - Naturaleza. Con el fin de llevar a cabo los objetivos mencionados, se presentará como estudio empírico el caso del Movimiento Binacional de Afectados ante el Plan de Terminación de la represa hidroeléctrica argentino-paraguaya Yacyretá, el cual cuenta con financiamiento del Consejo Nacional de Investigaciones Científicas y Técnicas (CONICET) de la República Argentina. En el caso mencionado las herramientas conceptuales de SCOT resultan insuficientes para dar cuenta de la relación entre naturaleza y tecnología, ya que el énfasis puesto en superar el determinismo tecnológico discute solamente la vinculación sociedad - tecnología poniendo el acento en aspectos antropocéntricos que invisibilizan la complejidad de los conflictos entornos a los modos de habitar, cuyo entramado expresa ontologías relacionales políticas, donde la distinción occidental naturaleza - cultura, y su corolario prometeico, es puesta en discusión.

Palabras clave: Naturaleza - Tecnología - Constructivismo Social de la Tecnología - Ecología Política Latinoamericana

\begin{abstract}
In the present article will discuss the relationship between Technology and Nature, in the context of the Social Constructivism of Technology (SCOT) and will find the possible arguments to allow avoiding falling as much in the technological determinism as in the social determinism at the moment to think of this relation. Secondly, this research aims to take the tools coming from the Latin American Political Ecology (EPL), focusing on the developments made from the so-called "decolonial turn" in Latin American studies and "ontological turn" within anthropology, the which converge in the work of Arturo
\end{abstract}

\footnotetext{
${ }^{1}$ Universidad Nacional de Mar del Plata, Universidad Nacional de Misiones, CONICET. E-mail: ayelencavalli@gmail.com Ciudad: Buenos Aires.

2 Facultad de Ciencias Agrarias, Universidad Nacional de Mar del Plata E-mail: tomascarrozza@gmail.com Ciudad: Buenos Aires.
} 
Escobar, to reflect on the possibilities offered by them for a decolonial reading of the Technology Nature dichotomy.To carry out the objectives above, will present as an empirical study the case of the Binational Movement of Affected Persons by the Completion Plan of the Yacyretá ArgentineParaguayan hydroelectric dam. This results presented has financing from the National Council of Scientific and Technical Research (CONICET) of the Argentine Republic. In the case mentioned, the conceptual tools of SCOT are insufficient to account for the relationship between nature and technology, since the emphasis on overcoming technological determinism discusses only the relationship between society and technology. This framework emphasizes anthropocentric aspects that invisibilize the complexity of the conflicts around the ways of inhabiting, a structure that expresses political relational ontologies, where the Western distinction nature-culture, and its Promethean corollary, is put in a discussion.

Key words: Nature - Technology - Social Construction of Technology - Latin American Political Ecology

\section{Introducción}

En el presente artículo se abordará la relación entre Tecnología y Naturaleza, dentro del Constructivismo Social de la Tecnología (SCOT, por sus siglas en inglés - Social Construction of Technology), y se indagaran los posibles argumentos que eviten recaer tanto en el determinismo tecnológico, que objetiviza a la Naturaleza, como en el determinismo social, que la reduce a una construcción social.

En segundo lugar, esta investigación se propone tomar las herramientas provenientes de la Ecología Política Latinoamericana (EPL), focalizando en los desarrollos realizados a partir de los denominados "giro descolonial" en los estudios latinoamericanos y "giro ontológico" dentro de la antropologia, los cuales confluyen en el trabajo de Arturo Escobar, para reflexionar sobre las posibilidades que ofrecen las mismas para una lectura decolonial de la dicotomía Tecnología - Naturaleza.

Con el fin de llevar a cabo los objetivos mencionados, se presentará como estudio empírico el caso del Movimiento Binacional de Afectados ante el Plan de Terminación de la represa hidroeléctrica argentino-paraguaya Yacyretá, el cual cuenta con financiamiento del Consejo Nacional de Investigaciones Científicas y Técnicas (CONICET) de la República Argentina.

A partir del mismo se buscará generar un primer acercamiento y reflexionar sobre la capacidad de diálogo de los conceptos y su pertinencia para abordar compresivamente la relación entre Naturaleza y Tecnología. 


\section{El lugar de la Naturaleza en el Constructivismo Social de la Tecnología}

Los aportes de la Social Construction of Technology (SCOT), cuyos principales representantes son T. Pinch y W. Bijker (2008), plantean un modelo de análisis multidireccional posicionando lo sociotécnico como nivel de análisis, donde se expresan controversias por la construcción de significados artefactuales en disputa.

Los Grupos Sociales Relevantes (GSR), mediante la construcción compartida de significado, son los que definen qué problemas son principales y quienes, al mismo tiempo, habilitan posibles soluciones. A su vez, los autores advierten que dicha significación puede generar heterogeneidad en determinado grupo social que era considerado homogéneo, ya que éstos no son estáticos (KLINE Y PINCH, 1996). En consecuencia, desde el modelo multidireccional, luego de identificar los GSR se debe proseguir en una descripción detallada que permita definir el funcionamiento / no funcionamiento que el grupo atribuye a determinado artefacto tecnológico. En términos de Bijker (1995), el funcionamiento o no funcionamiento de un artefacto no se deriva de sus propiedades intrínsecas sino de su construcción social. Lo anterior habilita identificar los problemas, con sus posibles soluciones, que cada grupo construye en torno al artefacto. El nivel de conflictividad da cuenta de diversos grados de estabilización que sufren los artefactos, que difiere en cada grupo, cuando la flexibilidad interpretativa disminuye y los mecanismos de clausura son efectivos, generando la "desaparición" de problemas y el cierre de determinada "controversia tecnológica". Por último, se identifica una vinculación del artefacto tecnológico a un medio socio-político más amplio, ya que los significados dados a un artefacto tecnológico por diversos GSR se encuentran influenciados por normas $y$ valores que son parte de determinada situación politica y sociocultural.

En trabajos posteriores, Bijker (2008) reconoce la necesidad de complejizar su abordaje teórico por temor a quedar preso de la dicotomía tecnología - sociedad, procurando que su unidad básica de análisis no sea el artefacto sino el marco tecnológico (technological frame). De inspiración 
kuhniana, la categoría de marco tecnológico (MT) es descripta por el autor como una combinación de conceptos, teorias, técnicas, conocimientos tácitos y codificados, prácticas y metas, que se ponen en juego por una comunidad con el propósito de definir problemas / soluciones. Es decir, los marcos habilitan ciertas posibilidades y restringen la capacidad de acción de los actores, al tiempo que las nuevas interacciones entorno a los artefactos configuran nuevos MT.

Bijker busca distanciarse de sus predecesores del desarrollo tecnológico cuando enfatiza que el marco tecnológico no refiere al tecnólogo, sino a la tecnología. En segundo lugar, se aclara como rasgo distintivo de este concepto su aplicación a la interacción de varios actores, es decir, "no es una característica individual, ni tampoco una característica de sistemas o instituciones; los marcos se encuentran entre actores, no en los actores ni encima de los actores. En ese sentido, los marcos son similares a las redes de Callon" (BIJKER, 2008, P. 81) ${ }^{3}$

Los actores poseen diversos grados de participación en los marcos, al tiempo que pueden ser miembros de varios simultáneamente. Como se mencionó en párrafos anteriores, los miembros de los grupos sociales atribuyen diversos significados a los artefactos, que proveen de cierta gramática a los marcos tecnológicos, como consecuencia de una construcción de sentido compartido.

Siguiendo a Giddens, Bijker (1995) adopta una concepción relacional del poder, donde dominación y capacidad transformativa dan cuenta de dos lados del poder: la estructura y la acción. En la constitución de los marcos tecnológicos aparece una dimensión política, la cual es explicada por el autor mediante dos términos, que se encuentran vinculados: poder semiótico (estructura), asociada a una fijación de sentido en los procesos de clausura y estabilización de artefactos, y micropolíticas del poder (acción), confirmadas por determinadas estrategias problema-solución, teorias y prácticas habilitadas por el marco tecnológico hegemónico.

En un tercer nivel de análisis, encontramos los ensambles sociotécnicos, como nuevas unidades de análisis, dentro de los cuales se puede

3 Sobre la teoría del Actor-Red a la que alude Bijker ver Callon $(1995,1986)$. 
identificar la dinámica de diversos marcos tecnológicos, con el objetivo de brindar una explicación superadora del modelo lineal de innovación que considera a la tecnología como mera ciencia aplicada.

A finales de siglo XX, el constructivismo social de la tecnología migra y se consolida como marco teórico predominante en el contexto de los estudios sociales de la ciencia y la tecnología en América Latina. En relación al abordaje de controversias tecnológico-ambientales ${ }^{4}$ dentro de la perspectiva SCOT, uno de sus principales exponentes, W. Bijker, ha realizado diversos casos de estudios empíricos, que se nuclean actualmente entorno a dos grandes temáticas: manejo de recursos hídricos y nanotecnología (BIJKER, 2011). En los trabajos realizados por Bijker (2005a, 2007a, 2007b) vinculados al manejo de recursos hídricos comparando casos en India, Estados Unidos y los Países Bajos. El autor reconoce la existencia de valores asociados a la democracia ambiental, relaciones de poder $\mathrm{y}$ políticas concretas. El estudio de este tipo de artefactos, en tanto socialmente construidos al tiempo que construyen la sociedad, tiene el objetivo de analizar las dimensiones culturales y democráticas del desarrollo socio-técnico de los denominados países del Norte y del Sur. A su vez, según el autor, categorías como "marco tecnológico" pueden ser útiles para una mayor comprensión de los conflictos generados por el manejo de recursos hídricos y el desarrollo de politicas de micro y macro escala que construyan prácticas locales de manejo hídrico democrático.

Asimismo Bjiker (2004) ha discutido sobre los debates públicos en torno al "desarrollo de la naturaleza" o "construcción de la naturaleza", políticas sustentables a partir de la experiencia de manejos hídricos en los Países Bajos y la participación de los ciudadanos "expertos" y "no expertos", que expresan distintos criterios de valoración en la construcción de los

\footnotetext{
4 Se puede mencionar como uno de los primeros antecedentes de abordaje de controversias tecnológico-ambiental los análisis realizados por Dorothy Nelkin (1979) sobre el movimiento antinuclear en Estados Unidos. Según Yearly (2008), desde los primeros estudios CTS, se evidenció que el enfoque de las ciencias ambientales resultaba estrecho para dar cuenta de la complejidad de la problemática. El medioambiente se posicionó como tópico central para comprender el status de lo natural en la modernidad avanzada y su vinculación con el desarrollo científico-tecnológico. Una de las preocupaciones centrales dentro de este campo gira en torno a la construcción discursiva de "la naturaleza" y "el medioambiente" en el marco de la dicotomía occidental naturaleza - cultura.
} 
ensambles sociotécnicos. El autor destaca la necesidad de que los abordajes de las ciencias ambientales amplien su horizonte disciplinar incorporando aportes del campo de estudios en Ciencia, Tecnología y Sociedad, tales como SCOT, con el objetivo de contribuir al desarrollo de políticas de manejo sustentable de los recursos naturales, entendidos como "procesos de aprendizaje abierto". Para dicho objetivo, Bjiker destaca que la aplicación de políticas ambientales exitosa implica una politización de la cultura tecnológica, que conduzca institucionales estatales sustentables estables.

Dichos trabajos muestran cierta distancia de los primeros análisis de SCOT sobre la baquelita y la bicicleta (BIJKER, 1995), que pareciera acusar recibo de algunas de las críticas recibidas desde la filosofia de la tecnología (Winner, 1993). En sus últimos estudios Bjiker destaca la relación entre tecnología y poder: cambiando el foco de lo micro a lo macro (Bijker, 2005b), enfatiza la necesidad de politicas públicas estatales que promuevan mayor democratización y procuren el desarrollo sustentable. La sustentabilidad como meta aquí es entendida en términos de un "desarrollo de la naturaleza" (Nature development), producto de la construcción social de la naturaleza. (BIJKER, 2004).

La perspectiva SCOT si bien es crítica del determinismo tecnológico parece mostrar, al menos, dos problemas que repercuten en el modo en que se piensa la relación entre tecnología y política: 1) las formas en que las posibilidades de acción técnica se habilitan y restringen no son explicitadas, de manera que, 2) se cae en una especie de determinismo social, donde el único aspecto que se enfatiza del vínculo es el rol de las relaciones sociales en conflicto, es decir, las relaciones de poder que configuran los diferentes grupos sociales relevantes cuando buscan posicionar su interpretación artefactual como dominante. Esto nos remite a otro punto que posee escasa articulación en la propuesta de Bijker: no se especifica la vinculación entre los niveles políticos micro de luchas interpretativas y el nivel macro del diseño de políticas públicas. El autor menciona a la tecnología como un medio para ampliar la participación en la arena política, particularmente de los llamados no-expertos técnicos, pero no explicita las mediaciones que 
permiten que la agencia de ciertos grupos sociales relevantes se traduzca en transformaciones de las instancias institucionales.

Si bien es posible visualizar un intento del constructivismo social de la tecnología por estudiar las controversias tecnológico-ambientales, se identifican ciertas limitaciones al momento de utilizar dicho marco teórico para investigar casos en la región que involucran la relación entre tecnología y naturaleza. Así, desde el constructivismo de la tecnología, la Naturaleza es ocultada, en tanto se la reduce a la narrativa y la construcción simbólica de "grupos sociales relevante". De esta forma, paradójicamente, con el objetivo de superar los determinismos tecnológicos, las dinámicas emergentes que surgen de la relación entre la Naturaleza y la Tecnología recaen en un determinismo social, que se expresa en perspectivas constructivistas radicales. Por otra parte, las discusiones en torno al desarrollo y al Estado Nación han recibido amplias críticas desde las experiencias latinoamericanas, a partir de categorías como "Buen vivir" y la constitución de Estados Plurinacionales.

Por lo anterior, a continuación, nos proponemos explorar los aportes que la Ecología Política Latinoamericana (EPL) a partir del giro descolonial, con el objetivo de indagar herramientas conceptuales que habiliten visibilizar y comprender con mayor profundidad y complejidad la relación entre naturaleza y tecnología.

\section{La Ecología Política Latinoamericana a partir del giro descolonial: La decolonialidad de naturaleza}

A finales del siglo XX un grupo de pensadores latinoamericanos conformaron un espacio de reflexión denominado Proyecto Modernidad/Colonialidad (M/C). Estos teóricos latinoamericanos plantearon una mirada transdisciplinaria y desjerarquizadora de saberes con el fin de reunir pensamiento crítico y praxis. El proyecto surge con la intención de establecer, a partir de una serie de conferencias en universidades estadounidenses y latinoamericanas, un diálogo entre los estudios sobre colonialidad y análisis del sistema-mundo de Immanuel Wallerstein. Sus raíces teórico-militantes son variadas y se encuentran en los debates y 
experiencias de los años setenta en América Latina motorizados por la teología y filosofia de la liberación, la teoría de la dependencia y la investigación-acción participativa; en los estudios poscoloniales y culturales anglosajones; en los debates neo-marxistas y posestructuralistas en Europa; en diversos feminismos y la filosofia afro-caribeña; entre otros (CF. ESCOBAR, 2003).

W. Mignolo (2007), sostiene que ha existido una crítica eurocéntrica de la modernidad que excluye su lado oscuro y constitutivo: la diferencia colonial, entendiendo modernidad y colonialidad como dos caras de una misma moneda. En la misma línea, A. Quijano (2007) explica como la expansión colonial de las potencias europeas en el siglo XV no solo implicó una dominación político, militar y económica del territorio sino también una colonialidad, es decir, relaciones de dominación y explotación que perduraron a nivel subjetivo, cultural y epistemológico, luego de la independencia politico-militar. Dicha colonialidad, entendida como eje de lucha, perspectiva y herramienta, se expresa, al menos, en cuatro áreas o ejes entrelazados: poder, saber, ser y naturaleza (WALSH, 2008)

Según Quijano (2007), la colonialidad del poder da cuenta del hecho de que el desarrollo de la modernidad implicó una forma de producción de conocimiento que configuró un patrón mundial de poder colonial/moderno, capitalista y eurocentrado. Es decir, existe una "geopolitica del conocimiento", en términos de Mignolo, que devela que el conocimiento no es abstracto ni deslocalizado, sino una manifestación de la diferencia colonial, y que se posiciona en diversos lugares epistémicos, éticos y políticos de enunciación. El desarrollo de la ciencia moderna, dado en Europa en el siglo XVII, posicionó al método científico como el único camino legítimo para llegar al conocimiento verdadero, generando una colonialidad del saber caracterizada por la supremacía gnoseológica de una razón instrumental. Las ciencias modernas, cuyo modelo por excelencia ha sido la fisica, transformaron a la naturaleza en un objeto de estudio regido por regularidades expresadas en leyes invariables y universales. La naturaleza es la otredad, una otredad que necesita ser domesticada, dominada, del 
mismo modo que el hombre racional domina su naturaleza interna, sus pasiones.

Para ello, fue necesaria la subordinación del cuerpo a la razón y, mediante las teorias científicas de la raza, el cuerpo se transformó en objeto de conocimiento, en tanto se lo objetivó como naturaleza. Asimismo, el ego cogito cartesiano muestra el privilegio que la modernidad le otorga al problema del conocimiento, por lo que considerar a los pueblos conquistados en América como inferiores cognitivamente implicaba una negación ontológica: cogito ergo sum toma la forma colonial de "en tanto Otros no piensan, no son". Aquí podemos dar cuenta de la colonialidad del ser, sustentada en el establecimiento de un sistema de clasificación social basado en una jerarquía racial y sexual de identidades homogéneas, negativas y esencialistas, cuyo antecedente a su expresión biologicista se encuentra en los debates entre Bartolomé de las Casas (1474 o 1784-1566) y Juan Ginés de Sepúlveda (1490-1573). A su vez, el ego conquiro dusseliano, fundamento práctico del 'yo pienso' (Dussel, 1996), es constitutivamente un ego fálico (Maldonado Torres, 2005; Lugones, 2011), que muestra la cercanía hacia la naturaleza que las mujeres ocuparon por ser consideradas racionalmente inferiores (Cavalli y Meske, en presa).

Desde su conquista América Latina se insertó en la división internacional del trabajo y la naturaleza se transformó en recurso natural para el desarrollo capitalista europeo. Al mismo tiempo, desde la concepción universal, evolucionista y eurocéntrica de la historia los pueblos colonizados fueron considerados incivilizados y pre-modernos. El origen del mito fundacional europeo del Estado moderno, el estado de naturaleza, fue vinculado a la clasificación racial de las sociedades no europeas. La dicotomía moderna naturaleza - sociedad colocó al no-europeo dentro del territorio pre-social, irracional e infra-humano.

En suma, se puede ver como las diversas manifestaciones de la colonialidad se ven entrelazadas, conformando una trama en la cual la colonialidad de la naturaleza ha tenido un lugar central en la configuración de la modernidad y del desarrollo capitalista. Según Escobar (2003), la 
naturaleza se presenta como "el otro lado de la diferencia colonial, con ciertas naturalezas - naturalezas coloniales/tercermundistas, cuerpos de las mujeres, cuerpos oscuros-, localizadas en la exterioridad de la Totalidad del mundo eurocéntrico masculino". Desde esta perspectiva, la crisis ambiental contemporánea es un emergente de la crisis de la modernidad eurocéntrica, la cual se expresa en un "régimen de naturaleza capitalista" con el propósito de subalternizar las expresiones locales de lo natural.

\section{Buen vivir como alternativa al desarrollo}

En contraposición a la colonialidad, Mignolo sostiene que hay una epistemología fronteriza, la cual "trabaja en el límite de los conocimientos indígenas subordinados por la colonialidad del poder, marginados por la diferencia colonial y los conocimientos occidentales traducidos a la perspectiva indígena de conocimiento y a sus necesidades politicas y concepción ética" (WALSH, 2002, P. 27-28). Esto conlleva la necesidad de construir nuevas genealogías conceptuales que den cuenta de la diferencia epistémica colonial y las geopolíticas del conocimiento, que habilite la posibilidad de auto-comprensión de los pueblos indígenas en América Latina, así como también de otras subjetividades negadas. Desde este posicionamiento, la otredad epistémica no constituye una exterioridad al pensamiento eurocéntrico ni tampoco formas de sincretismo o asimilación, sino que irrumpe en el intersticio y se expresa en términos de una corpopolítica del conocimiento que llevan adelante negros, trabajadores y mujeres, entre otros (CF. CASTRO-GÓMEZ Y GROSFOGUEL, 2007).

Diversas comunidades y movimientos sociales emergen con prácticas descoloniales. Particularmente, en los últimos años ha sido relevante en América Latina la irrupción de disputas territoriales que plantean nuevas formas de apropiación simbólica y material de la naturaleza. Estas luchas interpelan el ideario de la modernidad eurocéntrica, haciendo foco en uno de sus corolarios principales: el desarrollo.

A partir del trabajo de pensadores como Escobar (2010), Alimonda (2011), Gudynas (2011) y Leff $(1998,2003,2005)$, actualmente, la Ecología Política Latinoamericana (EPL) se ha posicionado como un espacio de saber 
que interpela el lugar que ha ocupado la naturaleza en el desarrollo del capitalismo y la modernidad eurocéntrica. Al mismo tiempo, se presenta como un conocimiento situado, ya que intenta recuperar, en clave descolonial, las experiencias y saberes no académicos que emergen en las diversas disputas ${ }^{5}$.

La Ecología Política es un campo interdisciplinario que emergen en los años setenta con el objetivo de problematizar el carácter político de un ámbito de saber que primero fue exclusivo de las ciencias naturales y, luego, objeto de indagación de la economía ambiental a partir de la crisis del petróleo y los "límites al crecimiento". Escobar (2010) sostiene que es posible describir a grandes rasgos tres generaciones dentro de la Ecología Política, se presentan dicha clasificación solo a modo panorámico, sin desconocer los matices y diferencias que pueden señalarse, dado que nos concentraremos en la posiciones contemporáneas:

a) Preconstructivista (años setenta) Corrientes de Economía ecológica criticas de la ideas neoclásicas, que entienden a la naturaleza como externalidad, y explicitan el carácter político de los conflictos ambientales.

b) Constructivista (años ochenta). Se registra una gran influencia del postestructuralismo, postmarxismo, postcolonialismo y fuerte carácter interdisciplinario, que pone en diálogo aportes de la geografia, antropología, ecologia, economía ecológica, historia ambiental, ecología histórica, estudios del desarrollo, estudios sociales de ciencia y tecnología. A nivel teórico se destacan posiciones antiesencialistas y constructivistas, al tiempo que se incorporan lecturas des la teoría liberal, marxismo, post-estructuralismo, teoria feminista, fenomenologia, teoría post-colonial, complejidad, y acercamientos de la ciencia natural tales como ecología del territorio y biología de la conservación.

c) Postconstructivista. Tendencia contemporánea de categorias emergentes como ontologías políticas y relacionalidad, que muestran la

\footnotetext{
5 Cabe señalar que es posible encontrar antecedentes sobre discusiones planteadas por la EPL en torno a la "colonialidad de la naturaleza" en pensadores latinoamericanos anteriores, tal como es el caso de Mariátegui, ampliamente estudiado por Alimonda (2007), y los escritos decimonónicos del pensador chileno Francisco Bilbao (Cavalli, 2018).
} 
convergencia del "giro descolonial" en el pensamiento latinoamericano y el "giro ontológico" en la antropología.

Actualmente, desde la EPL presentada se puede sostener que: "los debates sobre el posdesarrollo, el conocimiento local y los modelos culturales de la naturaleza han tenido que enfrentar (la) problemática del lugar. (...) las teorías del posdesarrollo y la ecología política son espacios esperanzadores para reintroducir una dimensión basada en el lugar." (ESCOBAR, 2000, P. 68). El "lugar", asociado a lo local, se contrapone al "espacio", en tanto construcción capitalista que se referencia con lo global. Desde esta perspectiva, la racionalidad del posdesarrollo se opone a una valorización capitalista y científica de la naturaleza y se expresa en movimientos sociales que defienden cuatro derechos fundamentales: 1) identidad, 2) territorio, 3) autonomía política, 4) visión propia del desarrollo.

Según Gudynas (2011), una buena parte de las críticas al desarrollo se han encausado en las propuestas presentadas bajo los conceptos en construcción de "buen vivir" (Ecuador) o "vivir bien" (Bolivia). La crítica al desarrollo se fundamenta en la visión antropocéntrica moderna que plantea un criterio de valoración utilitarista de la naturaleza. En contraposición, las expresiones como sumak kawsay del kichwa de Ecuador o suma qamaña del aymara de Bolivia, expresadas en las Reformas constitucionales de Ecuador (2008) y Bolivia (2009), reinterpretan el lugar de la naturaleza a partir de saberes indígenas. El "buen convivir" aymara "se lo vincula directamente a una vivencia plena, austera pero diversa, que incluye tanto componentes materiales como afectivos, donde nadie es excluido" y no existe una relación dual entre sociedad y naturaleza (GUDYNAS, 2011, P. 462). En caso boliviano encontramos la categoría ligada a una posición ético-moral vinculada al carácter plurinacional de la constitución y el cuidado de la naturaleza se restringe al derecho de tercera generación de los ciudadanos bolivianos a un ambiente sano. Mientras que en el texto ecuatoriano la naturaleza se presenta como sujeto de derecho. En la práctica, estos posicionamientos jurídicos entran en tensión con las estructuras económicas agroexportadoras de dichos países, como se muestra en el caso de la minería. 
Los casos mencionados, juntos otras luchas en la región, muestran la posibilidad de reivindicar pluralidad de saberes y proponer, en palabras de Leff $(1998,2005)$, racionalidades alternativas: la racionalidad ambiental en contraposición a la racionalidad instrumental. Consecuentemente, desde esta mirada la ecología política implica una epistemología política, que se nutre de diversas disciplinas y saberes.

Alimonda (2011) plantea que aquellos procesos de subordinación de América Latina al capitalismo como proveedora de materia prima implicó una perdida de la biodiversidad por el desarrollo de economías basadas en el monocultivo. Esta organización económica pensada siglos atrás para el beneficio de las metrópolis europeas persiste hoy en gran parte de los países de la región. Se plantea frecuentemente una falsa dicotomía entre economía y medioambiente, que expresa la afirmación de que los problemas ambientales son una preocupación exclusiva de los países industrializados, mientras que en la región la prioridad es superar el subdesarrollo (aun que ello implique muchas veces flexibilidad ambiental e impositiva para mantener puestos de trabajo).

\section{Ontologías políticas, relacionalidad y modos de habitar}

Por su parte, con el fin de cuestionar los dualismos ontológicos de la tradición racionalista occidental, Arturo Escobar (2013) recurre al marco fenomenológico de la cognición desarrollado por Maturana, Varela y Flores, y propone como superadora de dicha dicotomía a la noción de "relacionalidad" u "ontologías relacionales". El autor sostiene que "una ontología relacional es aquella en la cual nada preexiste a las relaciones que la constituyen. En estas ontologías, la vida es interrelación e interdependencia de cabo a rabo, siempre y desde el comienzo" (ESCOBAR, 2013, P. 35, cursivas en el original).

Asimismo, Escobar recupera la propuesta de antropólogos del denominado "giro ontológico". Con el objetivo de desarrollar metodologías etnográficas críticas al materialismo cultural que pudieran describir el modo de vida los achuar y awareté del Amazonia, los trabajos de campo de Phillip 
Descola (1986) y Eduardo Viveiros de Castro (1992, 2010) fueron referentes dentro del denominado giro ontológico de la antropología, desarrollando la postulación del "animismo" y del "perspectivismo multinaturalista" como ontologías amerindias.

En términos generales, este giro implicó dos cuestiones centrales: en primer lugar, una ruptura metodológica que invirtió la relación entre teoría y práctica, que pretendía evitar el clásico acercamiento al trabajo de campo con un marco teórico de categorias binarias eurocéntricas que debían ser verificadas con los datos extraídos de la experiencia etnográfica (HOLBRAND, 2010). En segundo lugar, se puede mencionar la superación de la escisión entre las etnografias orientadas a las formas de organización social y aquellas focalizadas en aspectos cosmológicos, la cual trajo como consecuencia una falsa oposición entre las dimensiones ontológicas y politicas de las comunidades estudiadas. Ambas cuestiones visibilizaron los sesgos ético-políticos que habían marcado un neocolonialismo eurocéntrico en términos metodológicos y la necesidad de redefinir categorias como “ontología” y "política” para poder dar cuenta de los modos de vida, es decir de las relaciones socio-politicas, entre existencias humanas y no humanas. La política se entiende como diferencias de poder y la ontología como los poderes de la diferencia (HOLBRAAD, MORTEN Y VIVEIROS DE CASTRO, 2013).

Descola se distancia de las posiciones clásicas que caracterizan a los sistemas animistas como aquellos donde plantas y animales se utilizan como metáforas del mundo social. Cada ontología prefigura una modalidad de colectivo, que se considera mucho más amplia que un "sistema social". Aquí la categoría "colectivo" es usada en el sentido dado por Bruno Latour en su teoría del Actor-Red, que conforman un "tejido sin costuras" de humanos (actores) y no-humanos (actantes) con capacidad de agencia (CF. LATOUR, 2008).

En la década del noventa, Descola redefine la ontología como: "la expresión concreta de cómo está compuesto un mundo particular, del tipo de equipamiento del que está hecho en función de la organización general especificada por un modo de identificación" (DESCOLA, 2014, P. 437). 
Identificación y relación son las dos modalidades fundamentales de estructuración de la experiencia individual y colectiva, es decir, se trata de un esquema general, "por medio del cual establezco diferencias y semejanzas entre otros existentes y yo mismo, al inferir analogías y contrastes entre la apariencia, el comportamiento y las propiedades que me adjudico y los que les atribuyo (...) Ese mecanismo de mediación entre un yo y un no-yo me parece, en el plano lógico, anterior y exterior a la existencia de una relación determinada." (DESCOLA, 2012, P. 177).

En la misma línea, Escobar también recupera el trabajo del antropólogo inglés Tim Ingold, quien describe en sus trabajos etnográficos, basándose en los escritos heideggerianos tardíos, una "ontología del habitar" en oposición a una "perspectiva del construir" hegemónica en el pensamiento occidental. Escobar (2013, p. 37) se pregunta “¿qué significaría desarrollar una práctica personal y colectiva de interser? ¿cómo innovamos con formas postdualistas de habitar el planeta...?"

Ingold entiende la perspectiva del habitar como aquella que "trata de la inmersión del organismo-persona en un ambiente o mundo de la vida como una condición de existencia ineludible" (INGOLD, 2000, P. 153). En oposición, la perspectiva del construir, "supone que la gente habita un mundo - o cultura o sociedad - al cual su forma y significado han sido ya adheridos. Se asume, en otras palabras, que se debe construir el mundo, conscientemente, antes de actuar en él (INGOLD, 2000, P. 153).

A su vez, es posible sostener que las diversas perspectivas mencionadas implican modos del habitar que entran en conflicto, el cual no es posible comprender desde lecturas reduccionistas e implica repensar la separación entre naturaleza y cultura, expresada paradigmáticamente en la dicotomía occidental naturaleza - tecnología (CAVALLI, 2017B). Los casos que a continuación se presentan constituyen experiencias exploratorias e intentos de abordajes compresivos desde la mirada de las ontologías políticas que se expresan en diversos modos del habitar.

\section{El caso del Movimiento Binacional de Afectados por la represa Yacyretá}


La represa Yacyretá es una iniciativa hidroeléctrica argentinoparaguaya cuya finalidad es lograr el aprovechamiento energético del Río Paraná. Si bien el Tratado firmado en 1973 dio comienzo al Proyecto, las obras de construcción se vieron dilatadas hasta mediados de los ochenta y principios de la década del noventa. El Plan de Terminación de Yacyretá se enmarcó dentro del Plan Energético Nacional 2004-2008, presentado por el presidente argentino Néstor Kirchner. La presidenta argentina Cristina Fernández de Kirchner realizó una extensión de dicho Plan, viéndose concluido éste el 12 de febrero del 2011, como resultado de la elevación de la cota del río a 83 metros sobre el nivel del mar y, consecuentemente, de la generación de energía al máximo nivel de potencia.

El 04 de mayo de 2017 se realizó la ceremonia de firma del Acta de Entendimiento tendiente a lograr el ordenamiento Económico y Financiero de la Entidad Binacional Yacyretá entre los presidentes Horacio Cartes de Paraguay y Mauricio Macri de Argentina. A partir de dicha Acta se planifica el desarrollo de otros proyectos binacionales: la ampliación de la represa Yacyretá, con la incorporación de 3 turbinas, y la colocación de 3 turbinas más en el brazo Aña Cuá. Además, se proyecta la construcción de la represa Itatí - Itá Corá sobre el río Paraná, descartando avanzar con el proyecto de Corpus.

El diseño y la implementación de las medidas contempladas dentro del Proyecto de Yacyretá, y del Plan de Terminación, en particular, han generado conflictos entre la Entidad Binacional Yacyretá, institución creada con el fin de implementar el Proyecto, y el Movimiento Binacional de Afectados por Yacyretá, compuesto por diversas organizaciones de comunidades afectadas de localidades argentinas y paraguayas, que nuclea a más de 80 mil grupos familiares. Las diversas agrupaciones que integran el Movimiento poseen una pluralidad de criterios de asociación y coordinación, entre ellos se destacan: actividades productivas, como oleros, pescadores, artesanos; pertenencia étnica, principalmente las comunidades guaranies; jurisdicción o barrios, y problemáticas específicas, entre ellas medioambientales, derechos humanos, etc. La heterogeneidad en la composición del Movimiento también se expresa en las diferentes 
perspectivas del conflicto y constituye un espacio complejo que habilita una pluralidad de criterios, saberes y experiencias (CAVALLI, 2011).

La terminación de las obras previstas en el Proyecto Yacyretá estaba supeditada a la concreción de medidas compensatorias de los perjuicios generados y por generar (Tratado Yacyretá, 1973, Artículos I, V, XVII, XVIII). Sin embargo, el proceso de toma de decisiones fue unilateral, sin participación efectiva de las comunidades afectadas y han sido inexistentes evaluaciones que den cuenta de los alcances reales de los impactos económicos, sociales, culturales y ambientales del proyecto.

El caso Yacyretá ha sido definido como un "Proyecto de Gran Escala" $\mathrm{y}$ se ha asociado a políticas que buscaban promover el progreso y el desarrollo regional (LINS RIBEIRO, 1999). Sin embargo, las relocalizaciones forzosas han generado un proceso de elitización del espacio urbano y "periferización" de los sectores populares (BRITES, 2014). Al tiempo que los afectados sufrieron una doble exclusión: perdida territorial y destrucción de las redes sociales (BRITES, 2010; BRITES Y CATULLO, 2015)

Las diferentes organizaciones del Movimiento han articulado estrategias de lucha en las cuales han sido relevantes las alianzas realizadas con otros sectores, como Organizaciones No Gubernamentales ambientalistas; universidades; medios de comunicación alternativos; cooperativas; organismos internacionales; entre ellos el Banco Mundial, el Banco Interamericano de Desarrollo y la Corte Interamericana de Derechos Humanos. La estrategia de la EBY ha sido intentar desarticular la capacidad de negociación colectiva de las organizaciones. Esta cuestión queda en evidencia cuando la EBY establece, en mayo del 2012, que los reclamos por las afectaciones generadas por Yacyretá solo pueden ser realizados de forma individual.

Por otra parte, el reclamo solamente es habilitado para aquellos que gozan del estatus de "afectado", luego reemplazado por la categoría de "beneficiario". El movimiento reconoce más de 80 mil núcleos familiares afectados en ambos países, que incluyen varias generaciones desde el comienzo de las obras en la década del ochenta hasta la fecha. Los listados 
oficiales de afectados por la zona del embalse que ha realizado la EBY no contaron con las actualizaciones necesarias, descartando afectados de segunda y tercera generación, ni contemplaron los afectados indirectos, es decir aquellos que no se encontraban viviendo en la zona que debía ser inundada, pero que su subsistencia dependía de algún modo de su cercanía al río (Cavalli, 2017). Por lo cual, la lucha por el reconocimiento del estatus de "beneficiario" se transformó en la única posibilidad de acceso a los beneficios de indemnización económica o relocalización ${ }^{6}$.

\section{Una lectura de la controversia tecnológico-ambiental desde la perspectiva SCOT}

Desde la perspectiva planteada en el apartado anterior, la represa hidroeléctrica Yacyretá puede ser entendendida como un artefacto, entorno a cuya construcción diversos Grupos Sociales Relevantes (GSR) atribuyen significados que dan lugar a diferentes concepciones de funcionalidad/no funcionalidad de dicho artefacto $\mathrm{y}$, consecuentemente, a la existencia de problemas y soluciones (CAVALLI, 2014).

Un grupo social relevante que se puede identificar es la Entidad Binacional Yacyretá (EBY), compuesta por directores que representan los interesen de ambos países, por lo cual, constituye un grupo social heterogéneo. Para el gobierno paraguayo, la generación de energía eléctrica de Yacyretá, al igual que en el caso de la represa Itaipú, tiene la finalidad de obtención de ganancias por exportación de energía a Brasil y Argentina, principalmente. En el caso argentino, el gobierno argumentó la necesidad de incremento en la generación de energía eléctrica con fin de abastecer a la industria en un contexto de escasez dado por la crisis energética mundial y la reactivación productiva argentina (CAMERON, 2004). Mas allá de las diferencias establecidas, ambos países acuerdan en atribuir una

6 En mayo de 2016 la Asamblea de afectados por Yacyretá por lo Derechos humanos el Medioambiente realiza una asamblea binacional en Posadas, Misiones, con el fin de exigir que la EBY cumpla con lo dictaminado por la Justicia argentina, la cual "dio lugar a la comunicación al Tesoro Nacional argentino que somos 80 mil afectados y el módulo de la demanda son 30 mil millones de dólares", sostiene Brígido Olivera, represente de la Asamblea. Sin embargo, este avance fue restringido por la falta de reconocimiento del EBY de gran número de organizaciones de afectados. A fines de mayo del 2016, Yacyretá ajusta listado de afectados, ratificando solamente unas 15 organizaciones de más de 60 , mientras que otras 15 organizaciones fueron dadas de baja y analizan la situación de unas 30 más. Ver: https://misionesplural.net/2016/05/31/yacyreta-ajusto-su-listado-de-afectados-por-larepresa/ 
significación economicista a la construcción de la represa. Para este GSR la funcionalidad del artefacto está dada por la necesidad de elevar la cota del río a $83 \mathrm{msnm}$, con el fin de que la generación de energía eléctrica alcance su máximo potencial, y así poder obtener los beneficios económicos esperados. Por lo tanto, la EBY ha identificado los obstáculos a la finalización de la construcción de la represa como un problema, ante el cual ha presentado el PTY como solución.

En segundo lugar, otro GSR son los organismos internacionales de crédito que financiaron y monitorearon las obras en torno a la construcción de la represa. La financiación del Proyecto Yacyretá provino de una serie de préstamos del Banco Internacional de Reconstrucción y Fomento (BIRF) y el Banco Interamericano de Desarrollo (BID) otorgados a la República Argentina. Dentro del Proyecto se incluyeron la construcción de la represa hidroeléctrica, la instalación de turbinas, expropiación de tierras, reasentamiento de familias, y mitigación y compensación ambiental. Uno de los objetivos importantes del Banco fue fomentar la inversión privada en el proyecto. Este GSR entendía a Yacyretá como la solución a los problemas de navegabilidad del río Paraná, riego de los cultivos de la zona, las crecidas del río y abastecer la necesidad de energía eléctrica. El problema fundamental en torno al artefacto fue los obstáculos al plan de implementación del proyecto que permitiera su finalización, causados por la incapacidad de la EBY para hacer frente a factores exógenos y endógenos de crisis. Consecuentemente, se puede afirmar que este GSR estableció alianzas con otro GSR, la EBY. En el marco del Plan de Terminación de Yacyretá, estos Bancos financiaron, por medio de préstamos al gobierno argentino, las obras necesarias para su implementación final (BID, 2004; BANCO MUNDIAL, 1999, 2002; QUINTERO SAGRE, 1992).

Por otra parte, se debe mencionar al Movimiento Binacional de Afectado por Yacyretá como GSR central en las controversias generadas por el Plan de Terminación de Yacyretá. Este grupo se caracteriza por una alta heterogeneidad en su composición, pero han logrado consensuar objetivos y estrategias de lucha, que incluyeron el "enrolamiento", en términos 
latourianos, de otros grupos sociales como universidades nacionales y ONGs ambientalistas. Para este GSR la significación atribuida al artefacto era la destrucción de sus formas de vida. La construcción de la represa constituía el problema de la destrucción de la naturaleza, la cultura y las formas de organización económica que tradicionalmente las comunidades relocalizadas habian tenido. Ante dicha problemática, la solución para el MBAY era impedir la elevación de la cota del río a $83 \mathrm{msnm}$ prevista en el Plan de Terminación de Yacyretá. Esta situación generó la Declaración de Posadas (2002), donde la construcción de la represa Yacyretá fue condenada por la Red Latinoamericana contra las Represas y por los Ríos, sus comunidades y el agua.

Sin embargo, como se ha mencionado, la heterogeneidad dentro del grupo mostraba matices en la definición de los problemas/soluciones vinculados al artefacto: algunos sectores veían como problema la ausencia de mecanismos de relocalización e indemnización económica adecuados. Al mismo tiempo, dicho grupo reclama la participación en los procesos de toma de decisiones y en el diseño del artefacto, en tanto solución a los problemas generados, resignificando el artefacto como "propiedad social".

Mientras que otros grupos, como el de las comunidades de pueblos originarios, atribuian al artefacto problemas de orden mas amplio que incluía lo moral, comunitario, emocional, religioso, etc., cuya solución no es posible mediante compensaciones económicas. La existencia de la represa es incompatible con sus formas de vida.

Otro Grupo Social Relevante que se debe mencionar esta compuesto por diversas Universidades Nacionales, que consensuaron espacios de lucha con el MBAY, ONGs, etc. En este GSR participaron grupos de investigación que se encontraban trabajando los problemas ambientales como consecuencia de la construcción de la represa (BLANCO ET AL, 2003; CANZIANI ET AL, 2003). Asimismo las ONGs ambientalistas constituyen un GSR, particularmente se destaca la participación en la controversia de la Fundación Vida Silvestre Argentina, la cual realizó un informe sobre el impacto ambiental de la Represa Yacyretá (BERTONATTI ET AL, 1993) que posteriormente fue ampliado. Estos dos últimos GSR, sin desconocer otras 
dimensiones de la controversia, enfatizan la destrucción del ecosistema como principal significado atribuido a la construcción de la represa. Para estos grupos, la solución no puede ser entendida como compensación económica $\mathrm{y}$, al igual que las comunidades de pueblos originarios que integran el MBAY, la existencia de la represa es incompatible con la conservación del ecosistema en riesgo.

A partir de la identificación y descripción de los Grupos Sociales Relevantes, se manifiesta la diversidad de significaciones atribuidas al artefacto. Dicha heterogeneidad se ha demostrado aun al interior de los GSR, como se documentó en el caso del Movimiento de afectados, y da cuenta de la flexibilidad interpretativa del artefacto: represa hidroeléctrica Yacyretá. Tal como Pinch y Bijker sostienen en relación a otros casos empíricos, esta flexibilidad se visualiza en la construcción e interpretación cultural que los diferentes Grupos Sociales realizan en torno a la represa y en la manera en que es planteado el diseño del artefacto.

Esta heterogeneidad genera controversias entre los grupos que son resueltas a partir del proceso de clausura y estabilización del artefacto. El GSR EBY, que representa la acción de los gobiernos de Argentina y Paraguay, logró posicionar su interpretación y significación del artefacto, colocando como problema la escasez de energía y su necesidad para reactivación productiva, cuya solución viable fue el Plan de Terminación de Yacyretá que elevó la cota del río Paraná a 83 metros sobre el nivel del mar.

Desde la perspectiva SCOT la naturaleza queda de alguno modo subsumida a la artefactualidad y las formas hegemónicas de gestión tecnológica, la cual es definida por la construcción simbólica de los GSR que lograron la clausura interpretativa del artefacto. De este modo, si bien se produce un alejamiento de posiciones naturalistas que objetizan a la naturaleza, un constructivismo radical que la reduzca a la discurisividad de los GSR corre el riesgo de caer un determinismo social que niegue la existencia de cualquier dimensión material de la naturaleza.

Por otra parte, tal como sostiene Quintanilla, la flexibilidad interpretativa de los artefactos no es ilimitada y está restringida por la 
materialidad de los mismos. En relación al clásico caso de estudio trabajado de Pinch y Bijker, Quintanilla sostiene que: "una bicicleta se puede ver como un instrumento de paseo o de competición, pero no sería técnicamente viable una interpretación que viera en ella un instrumento para freír patatas, para escribir cartas o para asar manzanas" (QUINTANILLA, 1998).

A continuación buscaremos realizar lecturas del caso presentado que incorporen miradas mas integrales y muestren la complejidad de la relación entre naturaleza y tecnología, incorporando herramientas de la EPL.

\section{Las comunidades Mbya Guaraní afectadas y el "Buen vivir" como crítica de la dicotomía Naturaleza - Técnica}

Un punto relevante que no fue contemplado en el diseño de las medidas de compensación, y representa un gran fracaso actualmente, es la situación de la relocalización de los pueblos originarios, con la consecuente pérdida de un patrimonio cultural milenario que va desde prácticas cotidianas hasta yacimientos arqueológicos, pasando por la eminente destrucción de vidas que se perdieron ante la incapacidad de adaptación en los asentamiento construidos por la EBY. Tal es el caso del pueblo Mbya Guaraní que habitó ancestralmente la cuenca e islas del Paraná sur en Paraguay y Argentina (en los actuales departamentos de Itapúa y Misiones), quienes fueron relocalizados sin consultas ni indemnización alguna, violando tratados internacionales.

En septiembre de 2015 se realizó el IV Encuentro Continental de la Nación Guaraní en la comunidad de ka`a kupe de Ruis de Montoya (Misiones, Argentina), bajo el lema "Yvy maraê'y Tierra/territorio, Justicia y Libertad"7. Allí Participaron más de 600 delegados del Concejo Continental de la Nación Guaraní (CCNAGUA) de Argentina, Paraguay, Bolivia y Brasil, quienes presentaron el Manifiesto Guaraní.

Amparados tanto en su pertenencia ancestral al territorio y la legislación vigente, como el "Convenio 169 de la OIT", la "Declaración de la Naciones Unidas sobre los Derechos de los Pueblos Indígenas”, y en

\footnotetext{
7 Disponible en: http://encuentroccnagua.blogspot.com.ar/2015/09/manifiesto-del-iv-encuentro-del-
} concejo.html 
"Declaración Universal de los Derechos Humanos", el Consejo exige a los Estados mencionados que se ponga fin a las "las persecuciones, desalojos, asesinatos y ataques casi diarios a líderes indígenas", "fundamentado en un derecho inmoral de conquista”. A su vez, se reclama a Argentina y Brasil la realización de los relevamientos territoriales estipulados por las leyes vigentes y la promulgación de una Ley de propiedad indígena comunitaria

En dicho contexto, la Naturaleza ocupa un lugar central en la vida de la Nación Guaraní y, consecuentemente, en los reclamos en torno al territorio:

\footnotetext{
"Este CCNAGUA expresando el sentimiento de la Nación Guaraní, que es una sola gran nación más allá de las fronteras políticas que nos fueron impuestas, que siente como su raíz más profunda la relación con la naturaleza de la que somos parte como el pindo y el kochi'i, y que tiene mucho que brindar a la diversidad del pensamiento de la humanidad; con una concepción de la vida, la salud y la educación que debe ser tenida en cuenta por los gobiernos".
}

El progreso económico se presenta como disruptivo del equilibrio natural, expresado en las políticas económicas extractivistas y en proyectos de Mega-represas hidroeléctricas en la región. Al respecto de este último punto el Manifiesto se opone al proyecto de "encarcelar a los ríos entre paredes", haciendo alusión a los casos de las Megarepresas Corpus Christi, Garabi, Roncador, Itaipú y Yacyretá.

Los aspectos políticos ligados a la autodeterminación de los pueblos; el ámbito jurídico que garantice leyes de protección del territorio; la educación y la cultura enraizada en la lengua, la música y la danza; actividades productivas como la tarea que realizan artesanos, pescadores y agricultores; el cuidado del cuerpo y el espíritu mediante plantas medicinales; todo estos son ámbitos que en el pensamiento occidental aparecen escindidos entre sí y alejados de la naturaleza. En la cosmovisión del pueblo Mbya Guaraní dicha esferas se entrelazan generando una trama vital en la cual es imposible distinguir los limites entes naturaleza y cultura, naturaleza y sociedad. Esta visión holista puede ser expresada en el término 
"buen vivir". Jorgelina Duarte, mujer de la comunidad Mbya Guaraní, se refiere al buen vivir del siguiente modo:

\begin{abstract}
"Para mí el Buen Vivir es imposible pensarlo sin los territorios, porque (...) el comer bien, sentirse bien socialmente, mentalmente y todo eso depende del territorio, porque en el territorio yo encuentro la medicina, la tierra, el agua (...) el Buen Vivir no puede estar aislado de lo que son los pueblos originarios, nosotros creemos que es importante nuestra voz, nuestro aporte para transformar esta sociedad tan individualista y a veces tan invasora y consumista. El buen vivir no es solamente que yo tenga, va muy ligado con lo territorial, no es sólo paz y amor, sino que también tiene que ver con la lucha politica."8
\end{abstract}

Dentro de la filosofia del Buen vivir el territorio se define como: 1) nuestra casa, donde proyectos de gestión comunitaria sustentable favorecen el cuidado de la coexistencia de la vida; 2) nuestro cuerpo, colonizado como la tierra e invisibilizado por la violencia racista y sexista del patriarcado, bajo modelos de belleza y tratamientos médicos eurocéntricos; 3) aquello que viaja con cada persona, porque es más que el lugar físico, somos parte y no dueños de la tierra; 4) donde se concreta la libre determinación de los pueblos, pensando que el Buen vivir debe favorecer el co-habitar de diferentes pueblos, aunque ello entre en tensión constante con los intereses de las empresas y el Estado que procuran el mero crecimiento económico. ${ }^{9}$

El buen vivir pone en relieve las tensiones entre diferentes modos de la vida, pero esto no implica una subordinación ni dominación de una forma sobre las otras, sino una relación armónica que no desconoce sino celebra la diversidad. Esta búsqueda de equilibrio no niega las tensiones ni las relaciones de poder, por el contrario, implica un compromiso político: a nivel micro, en el desarrollo de proyectos comunitarios y formas de organización asamblearias, y a nivel macro, en la coordinación de demandas colectivas ante el Estado y las grandes empresas. Estas mismas tensiones que pueden ver al interior del Movimiento binacional de afectados por Yacyretá. Por

\footnotetext{
8 Jorgelina Duarte, mujer de la comunidad Mbya Guaraní, en un encuentro del Movimiento Laicrimpo Salud, realizado en Rosario (Argentina) en noviembre del 2016. En Revista Superficie. "Buen vivir según las mujeres de pueblos originarios: derecho, territorio, amor y lucha". 09 de Diciembre de 2016. Disponible en: http://revistasuperficie.com.ar/mujeres-originarias-derecho-territorio-amor-ylucha.html

9 "Anteproyecto de Ley por el Buen vivir". Mujeres originarias por el buen vivir. Disponible en: https://mujeresoriginariasporelbuenvivir.files.wordpress.com/2015/04/proyecto-ley-consejomujeres-originarias.pdf
} 
ejemplo, en el mismo documento donde la FEDAYIN defiende la propiedad privada ante la Comisión Interamericana de Derechos Humanos, apoya la lucha por la propiedad comunal de los pueblos originarios ${ }^{10}$.

A partir de los reclamos y testimonios de los afectados, se plantean los límites de la mensurabilidad utilitarista asociada al progreso económico ${ }^{11}$. Desde una perspectiva estatal, la EBY entiende a la Naturaleza como una canasta de recursos o materias primas necesarias para la generación de energía en pos del desarrollo económico y descarta, implícitamente, las implicancias culturales asociadas a los procesos productivos. Esto se encuentra asociado a la dicotomía Naturaleza Sociedad que se configura con el desarrollo de la Modernidad eurocéntrica. $\mathrm{El}$ estado de Naturaleza, en tanto mito fundacional de una sociedad europea contractualista, se presenta como aquello salvaje, animal, pre-social, irracional. A partir de la conquista de América, la Naturaleza se ubica como el ámbito de lo colonizable y domesticable por el hombre europeo, blanco, occidental y cristiano, al tiempo que puesta al servicio del desarrollo capitalista se transforma en materia prima para el desarrollo industrial. De este modo, la relación dicotómica Naturaleza - Sociedad encierra una separación ontológica, un espacio de construcción de una otredad infravalorada que debía ser sometida, ya sea como insumo productivo o como objeto de investigación científica.

10 "el trato social otorgado a los indígenas no es más que la comisión de un verdadero etnocidio habiendo condenado a estos grupos indígenas a su extinción al privársele y trastocársele el sistema de vida milenario que llevaban (...) Se ha violentado su derecho a la vida, a la tierra, a la integridad personal y espiritual, han sido destruidas para siempre sus vinculaciones con la tierra donde nacieron, legada por sus antepasados, con su sistema propio de subsistencia, a su espacio de vinculación con la naturaleza y celebración religiosa que marca su existencia, apartándolo de su hábitat que es el lugar donde estos pueblos han vivido desde tiempos inmemoriales, y que por su propia cosmovisión la asociación tierra-hombre es el lazo que permite la existencia del Pueblo Mby“a, en su cultura, la tierra no tiene precio, es la "madre tierra", es madre de la vida, por tanto innegociable, por lo que cualquier medida de compensación económica es insuficiente ante la pérdida de su cultura, y en estos casos los daños son no mitigables, ya que está en juego parte del patrimonio de la humanidad (...) La EBY, atentando contra la cultura aborigen, simplemente les obligó a trasladarse dándoles tierra, e interpretando la situación desde nuestra cultura occidental pagando un precio por la tierra, cuando que para los aborígenes la tierra no tiene dueño, la tierra es parte del ser del hombre" (OEA, CIDH, 2005, p. 11).

11 "En la mente indígena no entra que un río se desvie para ciertas cosas, para obtener beneficios económicos. Siento en el alma que es algo tan diferente cuando hablan del progreso, para el sentido Mbya guaraní es muy diferente (...) Lo que pasó con nuestra gente realmente no cabría palabras para decir. Desaparecieron los lugares. Tanto para vivir como para morir. Nosotros decimos que nos cercenaron el derecho de nacer como personas libres. Se puede nacer fisicamente pero...la cultura no se recupera." (Brígido Bogado, Mbya Guaraní entrevistado en Cavalli, 2011, pp. 155-156) 
La posición del Movimiento de Afectados por Yacyretá, específicamente como mayor énfasis en las comunidades Mby’a Guaraní, Makás, Tupí y Panambí Guaraní, mediante su propuesta del Buen Vivir, cuestiona la escisión y subordinación de la Naturaleza al servicio de un orden social y económico de explotación capitalista. El Movimiento se opone a la valoración economicista y antropocéntrica presente en el diseño de las políticas de la EBY, al tiempo que las interpela poniendo en la arena las dimensiones culturales y éticas y proponiendo nuevas relaciones con la Naturaleza:

\footnotetext{
"El tratamiento de la naturaleza como un recurso que adquiere valor sólo en la explotación para el crecimiento económico ha sido central al proyecto del desarrollo. Es también central a la crisis del desarrollo. (...) La relación de una cultura ecológica con la naturaleza (...) es principalmente ética." (MBAY, 2010)
}

Se destaca en el caso del Movimiento binacional de afectados por la represa hidroeléctrica Yacyretá la relevancia de la dimensión politica del habitar, a partir de la formas de resistencia a las relocalizaciones de las comunidades de afectados. Dicha dimensión es central en el proyecto del "buen vivir" y entraña la superación de la escisión entre lo natural y lo artificial. Hay una disputa por dos perspectivas distintas del habitar el territorio: 1) una que subordina el habitar al construir y entiende a este último como producto de una mera planificación racional de profesionales; y 2) otra vivencia del habitar que integra el construir, los considera fundamentales al cuidado de la tierra y forma fundamental del ser del hombre. Esta segunda expresión del habitar se enuncia en forma poética, no en lenguaje científico-técnico. Esto implica que el habitar del hombre en la tierra debe volver a su esencia originaria que se perdió por la dominación de la ciencia y la tecnología sobre la naturaleza, lo cual ha conllevado su destrucción (CAVALLI, 2017A).

\section{Palabras Finales}

En el presente artículo se ha abordado la relación entre naturaleza y tecnología a partir de dos casos de estudio desde el Constructivismo Social de la Tecnología y la Ecología Politica Latinoamericana. 
Particularmente, la perspectiva SCOT ha sido ampliamente utilizada en aquellos análisis que han involucrado la relación Tecnología - Sociedad. Sin embargo, la popularización académica de su uso, así como sus objetos de análisis han dado lugar a un conjunto de invisibilizaciones, entre ellas, se destaca la asociada a la comprensión de la Naturaleza en las controversias tecnológico-ambientales. Así, desde el constructivismo de la tecnología, la Naturaleza es ocultada, en tanto se la reduce a la narrativa y la construcción simbólica de "grupos sociales relevante". Paradójicamente, con el objetivo de superar los determinismos tecnológicos, las dinámicas emergentes que surgen de la relación entre la Naturaleza y la Tecnología recaen en un determinismo social, que se expresa en perspectivas constructivistas radicales.

En dicho marco teórico la naturaleza queda subsumida a la artefactualidad y las formas hegemónicas de gestión tecnológica, definidas por la construcción simbólica de los GSR que lograron la clausura interpretativa del artefacto. De este modo, si bien se produce un alejamiento de posiciones naturalistas que objetivizan a la naturaleza, un constructivismo radical que la reduzca a la discursividad de los GSR corre el riesgo de caer un determinismo social que niegue la existencia de cualquier dimensión material de la naturaleza. En términos de Vogel (1995), esta última posición negaría la existencia independiente de una "naturaleza extradiscursiva", interpretada por diversas corrientes como el "fin de la naturaleza" (KWIATKOWSKA, 2006). Dicho debate se enmarca entre dos polos existentes: realismo y constructivismo radical o hiperconstructivismo, los cuales intentan ser articulados desde distintas perspectivas teóricas contemporáneas (HANNIGAN, 2006).

Otro problema que se observa en el planteo de Bjiker es la falta de articulación entre los niveles políticos micro y macro, que vincule la capacidad de agencia de los diversos grupos sociales y la planificación de politicas públicas. Esta desarticulación entre niveles es también representada en los modos de conceptualizar a la naturaleza en el contexto 
de este marco teórico y sus implicancias en el diseño y gestión de las políticas públicas.

El ocultamiento de la naturaleza se genera mediante técnicas de instrumentalización que "desanaturalizan" a la naturaleza, ya sea mediante instrumental de laboratorio o instrumentos de políticas públicas. Esta desnaturalización representada como democratización permite una manipulación y apropiación que se expresa en cuestiones como el "dialogo democrático" o las políticas públicas de desarrollo sustentable para el manejo de los recursos hídricos.

En conclusión, la naturaleza queda subsumida a un mero recurso objeto de disputa de distintos grupos políticos perdiendo su materialidad y status propio extradiscursivo. Así SCOT en pos de salir del determinismo tecnología-sociedad pierde de vista un horizonte mas amplio: la relación naturaleza-tecnología, mito fundacional de la diferencia antropológica occidental.

En el caso presentado, las herramientas conceptuales de SCOT resultan insuficientes para dar cuenta de la relación entre naturaleza y tecnología, ya que el énfasis puesto en superar el determinismo tecnológico, que subordina los procesos sociales a los cambios tecnológicos y se ha expresado en versiones de corte tecnocráticas, genera una trampa: discutir solamente sociedad y tecnología pone el acento en aspectos antropocéntricos. En contraposición, una lectura descolonial de la dicotomía Tecnología - Naturaleza permite develar la complejidad de los conflictos entornos a los modos de habitar, cuyo entramado expresa ontologías relacionales políticas, donde la distinción occidental naturaleza cultura, y su corololario prometeico, es erosionado.

\section{Bibliografía}

ALIMONDA, H. La colonialidad de la naturaleza: una aproximación a la Ecologia Política Latinoamericana. En ALIMONDA, H. (Comp.) La Naturaleza colonizada: Ecología política y minería en América Latina. Buenos Aires: CLACSO / Ediciones Ciccus, 2011.

ALIMONDA, H. La ecología política de Mariátegui. Tareas, Panamá, n. 125, 2007. 
BANCO INTERAMERICANO DE DESARROLLO. Informe Final de la Comisión Investigadora del BID, Proyecto Hidroeléctrico Yacyretá 760/OC-RG. Banco Interamericano de Desarrollo, 2004. Disponible en: <http:/ /www.iadb.org/iim/pr191713esp.pdf>

BANCO MUNDIAL. International Advisory Panel Report on the ArgentinaParaguay Yacyretá Hydroelectric Project. Banco Mundial, 1999. Disponible en <http://www.inspectionpanel.org> y <http://www.worldbank.org/ar>

BANCO MUNDIAL. Respuesta ante la Solicitud $N^{\circ} \mathrm{RQ02} / 1$ presentada al Panel de Inspección. Argentina/Paraguay: Proyecto Hidroeléctrico Yacyretá (con el apoyo actual del préstamo BIRF 2854-AR). Banco Mundial, 2002. Disponible en: <http://www.inspectionpanel.org > y <http://www.worldbank.org/ar>

BERTONATTI, C.; BANCHS, R. La represa Yacyretá y su impacto ambiental. Diagnostico y recomendaciones. Bol. Técnico Fundación Vida Silvestre Argentina. Buenos Aires: Silvestre Argentina, 1993.

BIJKER, W. The Politics of Water - the Oosterschelde Storm Surge Barrier: A Dutch Thing to Keep the Water out or Not. EN: LATOUR, B.; - WEIBEL, P. (Ed.). Making Things Public: Atmospheres of Democracy. Cambridge, MA: MIT Press, 2005a.

BIJKER, W. E. ¿Cómo y por qué es importante la tecnología?. Redes, 11(21), 19-53, 2005b.

BIJKER, W. E. American and Dutch Coastal Engineering: Differences in Risk Conception and Differences in Technological Culture. Social Studies of Science. 37:143-151. London, Thousand Oaks CA, New Delhi: SAGE Publications, 2007a.

BIJKER, W. E. Dikes and Dams, Thick with Politics. Isis, 98, 109-123, 2007b.

BIJKER, W. E. La construcción social de la baquelita: hacia una teoría de la invención. En: THOMAS, H.; BUCH, A. Actos, Actores y Artefactos: sociología de la tecnología. Bernal: Universidad Nacional de Quilmes, 2008.

BIJKER, W. E. Of Bicycles, Bakelites, and Bulbs. Toward a Theory of Sociotechnical Change. Cambridge, Massachusetts, Londres: MIT Press, 1995.

BIJKER, W. E. Towards a Knowledge Commons by Recognizing the Plurality of Knowledge - Experiences with democratic governance of science and technology. IASC Conference in Hyderabad, India, 2011. Disponible en: <http: / / hdl.handle.net/10535/7299>

BIJKER, W. E.; HUGHES, T. P.; PINCH, T. F. The Social Construction of Technological Systems. Cambridge: MIT Press, 1987. 
BJIKER, W. E. Sustainable Policy? A Public Debate about Nature Development in the Netherlands. History and Technology, UK, v. 20, n. 4, pp. 371-391. Routledge, Taylor \& Francis Group, 2004.

BLANCO, D. E.; PARERA, A. F.; ACERBI, M. H. La inundación silenciosa. El aumento de las aguas en los Esteros del Iberá: La nueva amenaza de la Represa Yacyretá. (Versión ampliada y actualizada). Buenos Aires: Fundación Vida, 2003.

BRITES, W. Procesos socio-urbanos complejos. Hacia un abordaje multidimensional de la ciudad de Posadas, Argentina. EN: MILLAN y BRITES. Ciudades Vivas: Imaginaciones sobre el territorio. Posadas: Ed. Creativa, 2014.

BRITES, W. Transformación urbana y periferización. La Experiencia de los conjuntos habitacionales de población relocalizada. Revista del Instituto Universitario de Urbanistica de la Universidad de Valladolid, $n^{\circ}$. 13, pp. 219-237, 2010.

BRITES, W. Y CATULLO, M. R. Grandes proyectos hidroeléctricos. Un análisis de los efectos sociales y urbanos de las represas de Salto Grande y Yacyretá. XI Reunión de Antropologia del Mercosur, Montevideo, Uruguay, 2015. Disponible en: <http://xiram.com.uy/actas-del-congreso/grupos-detrabajo/ponencias-grupo-de-trabajo-82>

CALLON, M. Algunos elementos para una sociología de la traducción: la domesticación de las vieiras y los pescadores de la bahía de St. Brieuc. En IRANZO, J. M. et al. Sociologia de la ciencia y la tecnologia. Madrid: CSIC, 1995;

CALLON, M. The Sociology of an Actor-Network: the case of the Electric Vehicle. EN: CALLON, M.; LAW, J. Y.; RIP, A. Mapping the Dynamics of Science and Technology. London: MacMillan Press, 1986.

CAMERON, D. Plan Energético Nacional 2004-2008. Programa de Gestión. Presidencia de la Nación Argentina [online]. Ministerio de Planificación Federal, Inversión Pública y Servicios. Secretaría de Energía, 2004. Disponible en: <http://www.energia.gov.ar>

CANZIANI, G.; ROSSI, C; LOISELLE, S. Y FERRATI, R. Los esteros del Iberá. Informe del proyecto: " $\mathrm{El}$ manejo sustentable de Humedales en el Mercosur". Buenos Aires: Fundación Vida Silvestre Argentina, 2003.

CASTRO-GÓMEZ, S. Y GROSFOGUEL, R. E1 giro decolonial: reflexiones para una diversidad epistémica más allá del capitalismo global. Bogotá: Siglo del Hombre Editores, 2007.

CAVALLI, A. Crisis energética y formas de apropiación de la Naturaleza. El caso del Movimiento Binacional de Afectados por la represa hidroeléctrica Yacyretá. Tesis de Licenciatura en Relaciones 
Internacionales. Universidad Nacional del Centro de la Provincia de Buenos Aires, 2011.

CAVALLI, A. El problema de la descolonialidad de la Naturaleza en las raíces del pensamiento latinoamericano. En: PAOLICCHI, L. \& MESCHINI, P. (Comps). Discursos y politicas de la descolonialidad. Mar del Plata: EUDEM, 2018.

CAVALLI, A. Implicancias culturales y políticas de las Controversias Tecnológicas. Una lectura del Plan de Terminación de la represa hidroeléctrica Yacyretá desde la perspectiva del Constructivismo Social de la Tecnología. En: BARBOSA, Susana (Comp.) Trasmutaciones y usos de lo politico en la sociedad de hoy. Mar del Plata: Universidad Nacional de Mar del Plata y Universidad del Salvador, 2014.

CAVALLI, A. Lo natural y lo artificial en la perspectiva del habitar de Tim Ingold. XII Coloquio internacional de filosofia de la técnica. El estatuto de lo artifiicial. Mar del Plata: UNMDP. 18 al 20 de octubre de 2017. ISBN en trámite, 2017b.

CAVALLI, A. Naturaleza, Tecnología y Política. Una lectura desde los movimientos sociales". XII Reunión de Antropólogos del Mercosur. Experiencias etnográficas $y$ desafios para el siglo XXI. Posadas: Universidad Nacional de Misiones. 4 al 7 de diciembre de 2017. ISBN 978987-42-7173-0, 2017a.

CAVALLI, A. Y MESKE, V (en prensa). La subjetividad desde un lugar de enunciación latinoamericano. Fisuras de la modernidad eurocéntrica. En: SALERNO, G. Y ASSALONE, E. Siempre soy: Para una filosofia crítica del sujeto. Mar del Plata: EUDEM, en prensa.

DECLARACIÓN DE POSADAS. En Marco Global de Referencia para la Acción Jurídica en la Defensa del Medio Ambiente, los Derechos Humanos, Contra las Represas y otros megaproyectos neoliberales. Chiapas: CIEPAC, 2002.

DESCOLA, P. La composition des mondes. Entretiens avec Pierre Charbonnier. París: Flammarion, 2014.

DESCOLA, P. La nature domestique. Symbolisme et praxis dans l'écologie des Achuar. París: Maison des Sciences de l'Homme, 1986.

DESCOLA, P. Mas allá de naturaleza y cultura. Buenos Aires: Amorrortu, 2012 .

DOMÍNGUEZ, D., \& SABATINO, P. Con la soja al cuello: crónica de un pais hambriento productor de divisas: Los Tormentos de la Materia. Contribuciones para una Ecología Politica latinoamericana. Buenos Aires: CLACSO, 2006. 
DUSSEL, E. Filosofia de la Liberación. Bogotá: Nueva América, 1996.

ESCOBAR, A. Ecologias politicas postconstructivistas [online]. Disponible en:<http://www.unc.edu/ aescobar/text/esp/escobar.2010.EcologiasPolitic asPostconstructivistas.pdf $>$

ESCOBAR, A. El final del salvaje. Naturaleza, cultura y politica en la antropologia contemporánea. Bogotá: Instituto Colombiano de antropología, 1999.

ESCOBAR, A. El lugar de la naturaleza y la naturaleza del lugar: ¿globalización o postdesarrollo?. En: LANDER, E. (Comp.). La colonialidad del saber: eurocentrismo y ciencias sociales. Perspectivas Latinoamericanas. Buenos Aires: CLACSO, 2000. Disponible en: <http://bibliotecavirtual.clacso.org.ar/ar/libros/lander/escobar.rtf>

ESCOBAR, A. En el trasfondo de nuestra cultura: la tradición racionalista y el problema del dualismo ontológico. Tábula raza. Bogotá, n. 18, pp. 15-42, 2013

ESCOBAR, A. Mundos y conocimientos de otro modo. El programa de investigación de Modernidad/colonialidad latinoamericano. Tabula Rasa. Bogotá, Colombia, n.1, pp. 51-86, 2003.

GÓMEZ, M. Algunas contribuciones de Arturo Escobar a la ecología política. Ecologia Politica, n. 50, pp. 101-106, 2016.

GORDILlO, G. Los escombros del progreso. Ciudades perdidas, estaciones abandonadas y desforestación sojera en el norte argentino. Buenos Aires: Siglo XXI, 2018.

GRAS, C., \& HERNÁNDEZ, V. Modelos de desarrollo e innovación tecnológica: una revolución conservadora. Mundo Agrario, n. 17, v. 36, 2016.

GUDYNAS, E. Buen vivir: germinando alternativas al desarrollo. América Latina en Movimiento, N. 462, pp. 1-20; febrero 2011.

HANNIGAN, J. Environmental sociology. New York: Routledge, 2006.

HOLBRAAD, M. Against the motion. En: VENKATESAN, S (ed.) Ontology Is Just Another Word for Culture: Motion Tabled at the 2008 Meeting of the Group for Debates in Anthropological Theory, University of Manchester, 2010.

HOLBRAAD, M.; PEDERSEN, M. Y VIVEIROS DE CASTRO, E. The Politics of Ontology: Anthropological Positions. Cultural Anthropology, 2013. Disponible en: <http://culanth.org/fieldsights/462-the-politics-of-ontologyanthropological-positions > 
KWIATKOWSKA, T. Lo natural, un concepto enigmático. Ludus Vitalis, vol. XIV, num. 25, pp. 153-161, 2006.

LATOUR, B. Reensamblar lo social. Una introducción a la teoria del actor-red. Buenos Aires: Manantial, 2008.

LEFF, E. (Coord.). Ecologia y capital. Racionalidad ambiental, democracia participativa y desarrollo sustentable. $3^{a}$ Ed. México: Siglo XXI Editores, 1998.

LEFF, E. La ecología política en América Latina. Un campo en construcción. Texto elaborado y presentado en la reunión del Grupo Ecologia Politica de CLACSO, celebrada en la cuidad de Panamá, 2003.

LEFF, E. La geopolítica de la biodiversidad y el desarrollo sustentable. Economización del mundo, racionalidad ambiental y reapropiación social de la naturaleza. Observatorio Social de América Latina, VI (117), 263-273. CLACSO, 2005.

LINS RIVEIRO, G. La represa de Yacyretá. Capitalismo transnacional y politica hidroenergética en la Argentina. Posadas: Editorial Universidad Nacional de Misiones, 1999;

LUGONES, M. Hacia un feminismo descolonial. Revista La manzana de la discordia, n. 6, v. 2, pp. 105-119, 2011.

MALDONADO TORRES, N. Liberation Theology and the Search for the Lost Paradigm: From Radical Orthodoxy to Radical Diversality. En PETRELLA, I. (Ed.). Latin American Liberation Theology: The Next Generation. Maryknoll: Orbis Books, 2005.

MIGNOLO, W. El pensamiento decolonial: desprendimiento y apertura. Un manifiesto. En CASTRO-GÓMEZ, S. Y GROSFOGUEL, R. El giro decolonial: reflexiones para una diversidad epistémica más allá del capitalismo global. Bogotá: Siglo del Hombre Editore, 2007.

MOVIMIENTO BINACIONAL DE AFECTADOS POR YACYRETÁ. ¿Qué hacer? Responsabilidad de la $E B Y$ [online]. Movimiento Binacional De Afectados Por Yacyretá. Disponible en: <http://www.yacyreta.info>

NELKIN, D. Controversy: Politics of Technical Decisions. Beverly Hills: Sage Publications, 1979.

ORGANIZACIÓN ESTADOS AMERICANOS, COMISIÓN INTERAMERICANA DE DERECHOS HUMANOS. Denuncia ante la Comisión Interamericana de Derechos Humanos en contra del Estado de Paraguay. Presentada ante la Comisión Interamericana de Derechos Humanos. Denunciantes: Jorge Olegario Urusoff, Organización No Gubernamental Tayí (ONG Tayi), FEDAYIM (Federación de Asociaciones y Organizaciones de Afectados por la Represa de Yacyretá de Itapúa y Misiones), todos de la ciudad de 
Encarnación, Departamento de Itapúa, República del Paraguay. Disponible en: <http:/ / www.yacyreta.info>

QUIJANO, A. Colonialidad del poder y clasificación social. En CASTROGÓMEZ, S. Y GROSFOGUEL, R. El giro decolonial: reflexiones para una diversidad epistémica más allá del capitalismo global. Bogotá: Siglo del Hombre Editores, 2007.

QUINTANILLA, M. A Técnica y cultura. Revista internacional de filosofia Teorema, Vol. XVII/3 (otoño de 1998). Pp. 49-69, 1998.

QUINTERO SAGRE, J. D.; RODEROS, R. A.; CAMPOS CERVERA, E. Informe de evaluación ambiental. Proyecto Hídrico Yacyretá [online]. Banco Mundial, 1992. Disponible en: <http://www.worldbank.org>

THOMAS, H.; JUAREZ, P.; PICABEA, F. ¿Qué son las tecnologias para la inclusión social? Red de Tecnología para la Inclusión social. Universidad Nacional de Quilmes, 2015.

TRATADO YACYRETÁ. Tratado Yacyretá [online]. Yacyreta, 1973. Disponible en: <http: / / www.yacyreta.org.ar/docum/2006/tratadodeyacyreta.pdf>

VIVEIROS DE CASTRO, E. From the enemy point of view. Humanity and divinity in an Amazonian society. Chicago: University of Chicago Press, 1992.

VIVEIROS DE CASTRO, E. Metafísicas canibales: lineas de antropología posestructural, Buenos Aires: Katz ediciones, 2010.

VOGEL, S. The Concept of Nature in Critical Theory. New York: SUNY Press, 1995.

WALSH, C. Interculturalidad, plurinacionalidad y decolonialidad: las insurgencias político-epistémicas de refundar el Estado. Tabula Rasa. Bogotá - Colombia, n. 9, pp.131-152, julio-diciembre, 2008.

WALSH, C. Las geopoliticas del conocimiento y colonialidad del poder. Entrevista a Walter Mignolo. En: WALSH, C.; SCHIWY, F.; CASTRO-GÓMEZ, S. (Eds.) Indisciplinar las ciencias sociales. Geopoliticas del conocimiento y colonialidad del poder. Perspectivas desde lo andino. Quito: Universidad Andina Simón Bolivar, 2002.

WINNER, L. Upon opening the black box and finding it empty: social constructivism and the philosophy of technology. Science, Technology \& Human Values, Vol. 18, No. 3, Pp. 362-378, 1993.

YEARLEY, S. Nature and the Environment in Science and Technology Studies". En: HACKETT, E.; AMSTERDAMSKA, O.; LYNCH, M.; WAJCMAN, J. (eds.) The Handbook of Science and Technology Studies. Third Edition. Cambridge: The MIT Press, 2008. 